\title{
Intensity Interferometry of Single X-Ray Pulses from a Synchrotron Storage Ring
}

\author{
A. Singer, ${ }^{1, *}$ U. Lorenz, ${ }^{1, \dagger}$ A. Marras,${ }^{2}$ A. Klyuev, ${ }^{2}$ J. Becker,${ }^{2}$ K. Schlage,${ }^{1}$ P. Skopintsev, ${ }^{1,3}$ O. Gorobtsov, ${ }^{1,4}$ \\ A. Shabalin, ${ }^{1}$ H.-C. Wille, ${ }^{1}$ H. Franz,${ }^{1}$ H. Graafsma,${ }^{2,5}$ and I. A. Vartanyants ${ }^{1,6, \#}$ \\ ${ }^{1}$ Deutsches Elektronen-Synchrotron DESY, Notkestraße 85, D-22607 Hamburg, Germany \\ ${ }^{2}$ Center for Free-Electron Lasers, Notkestrasse 85, D-22607 Hamburg, Germany \\ ${ }^{3}$ Moscow Institute of Physics and Technology (State University), Dolgoprudny, 141700 Moscow Region, Russia \\ ${ }^{4}$ National Research Center "Kurchatov Institute," Kurchatov Square 1, 123182 Moscow, Russia \\ ${ }^{5}$ Mid Sweden University, S-851 70 Sundsvall, Sweden \\ ${ }^{6}$ National Research Nuclear University, “MEPhI," 115409 Moscow, Russia
}

(Received 9 February 2014; published 6 August 2014)

\begin{abstract}
We report on measurements of second-order intensity correlations at the high-brilliance storage ring PETRA III using a prototype of the newly developed adaptive gain integrating pixel detector. The detector records individual synchrotron radiation pulses with an x-ray photon energy of $14.4 \mathrm{keV}$ and repetition rate of about $5 \mathrm{MHz}$. The second-order intensity correlation function is measured simultaneously at different spatial separations, which allows us to determine the transverse coherence length at these x-ray energies. The measured values are in a good agreement with theoretical simulations based on the Gaussian Schell model.
\end{abstract}

DOI: 10.1103/PhysRevLett.113.064801

Intensity interferometry, pioneered by the experiment of Hanbury Brown and Twiss (HBT) [1,2], is an exemplary tool to investigate fundamental properties of partially coherent radiation. Originally designed as a robust setup to determine the size of stars, it led to the development of new concepts in statistical optics [3] and especially initiated research in the field of quantum optics [4]. Intensity interferometry revealed an important concept of photon bunching as a consequence of the boson statistics of photons, contrary to the antibunching observed for fermionic particles [5]. It also led to the discovery of fundamentally distinct statistics of thermal sources and lasers [6]. Over decades, HBT experiments have been used to study the coherence properties of thermal sources in a large spectrum ranging from visible light to $\mathrm{x}$-ray energies [7-10]. Recently, the possibility to perform intensity interferometry at free-electron lasers was demonstrated as well [11]. Such an approach is particularly attractive because in the HBT experiment intensities are measured; hence, it is not sensitive to phase fluctuations due to atmospheric turbulence or optics instabilities, which often compromise amplitude correlation methods, such as Michelson or Young's experiments $[12,13]$.

The key quantity in a HBT experiment is the normalized second-order intensity correlation function

$$
g^{(2)}\left(\mathbf{r}_{1}, \mathbf{r}_{2}\right)=\frac{\left\langle I\left(\mathbf{r}_{1}\right) I\left(\mathbf{r}_{2}\right)\right\rangle}{\left\langle I\left(\mathbf{r}_{1}\right)\right\rangle\left\langle I\left(\mathbf{r}_{2}\right)\right\rangle},
$$

where $I(\mathbf{r})$ is the intensity measured at position $\mathbf{r}$ and $\langle\cdots\rangle$ denotes the average over a large ensemble of independent measurements. Using the Gaussian moment theorem [13], it can be shown that the intensity correlation function
PACS numbers: 41.60.Ap, 41.50.+h, 42.25.Kb, 42.50.Ar

originating from thermal (chaotic) sources can be described in terms of the amplitude correlation function, also known as the complex coherence function $\gamma\left(\mathbf{r}_{1}, \mathbf{r}_{2}\right)$, as [14]

$$
g^{(2)}\left(\mathbf{r}_{1}, \mathbf{r}_{2}\right)=1+\zeta\left|\gamma\left(\mathbf{r}_{1}, \mathbf{r}_{2}\right)\right|^{2},
$$

where $\zeta=1 / M_{l}$ is the contrast value, $M_{l}=T / \tau_{c}$ is the number of longitudinal modes of the radiation field, $T$ is the time resolution or pulse duration for pulsed sources, and $\tau_{c}$ is the coherence time, which scales inversely with the bandwidth and can typically be tuned [15]. The modulus of the complex coherence function $\left|\gamma\left(\mathbf{r}_{1}, \mathbf{r}_{2}\right)\right|$ is a measure of the visibility of interference fringes in a Young's double pinhole experiment $[12,13]$.

Synchrotrons and free-electron lasers are nowadays the principal sources of high-brilliance hard x-ray radiation. They generate beams with a high degree of coherence that led to the development of advanced coherence based techniques, such as coherent diffraction imaging [16-18] and x-ray photon correlation spectroscopy [19]. With the ongoing development of coherent $\mathrm{x}$-ray sources, including diffraction limited ultimate storage rings [20], measurements of the transverse coherence properties of x-ray radiation become increasingly important and are vital for the success of coherence-based applications. These measurements can also provide important information on the source parameters, such as the photon source size, and can be used, in principle, in a feedback system.

Transverse coherence measurements based on well established methods of amplitude interferometry, such as Young's double-pinhole experiment, work well in the soft $x$-ray range [21-23] but are extremely challenging in the hard $\mathrm{x}$-ray range due to the high penetration depth of $\mathrm{x}$-ray 
radiation. Different approaches were used to determine the coherence properties of hard x-ray sources [24-26]; in particular, several groups developed intensity interferometers at synchrotron sources [8-10].

The key to the success of intensity interferometry at synchrotron sources was the development of highresolution monochromators and the use of avalanche photodiodes (APDs), which have sufficient temporal resolution to discriminate single synchrotron radiation pulses. However, because of the poor spatial resolution of APDs, a beam splitter in combination with well-defined slits was required to access the intensity correlations for only one pair of transverse coordinates within the coherence length of the beam. To map out the full transverse correlation function, the measurement had to be repeated multiple times.

In this work, the new adaptive gain integrating pixel detector (AGIPD) is used to measure intensity correlations. This detector is able to resolve individual synchrotron pulses and has an exemplary spatial resolution, which eliminates the necessity of a beam splitter. Additionally, it measures intensity correlations at different relative positions across the beam simultaneously, which allows full coherence characterization in a single measurement.

The AGIPD $[27,28]$ is a novel detector system designed for experiments performed at the European $\mathrm{x}$-ray freeelectron laser (XFEL) [29]. It is aimed towards the demanding requirements of this machine for two-dimensional (2D) imaging systems. AGIPD is based on a hybrid pixel technology and operates most effectively in the energy range between 3 and $15 \mathrm{keV}$. It employs an adaptive gain switching amplifier with several stages, which adjusts the gain based on the number of detected photons per pixel. The current design goals are a dynamic range of more than $10^{4}$ per pixel for $12.4 \mathrm{keV}$ photons in the lowest gain, single photon sensitivity in the highest gain, and, importantly for XFEL applications and for our HBT experiment, operation at a frame rate of multiple $\mathrm{MHz}$. Charges are stored in a memory bank inside each pixel and can be read out between the x-ray bursts, allowing one to effectively capture and read out several thousand frames per second.

For our experiment, we used an AGIPD 0.4 assembly, which is a $16 \times 16$ pixel prototype of the AGIPD [30]. A silicon sensor thickness of $320 \mu \mathrm{m}$ gave a quantum efficiency of approximately $44 \%$ at $14.4 \mathrm{keV}$ photon energy. The pixels are $200 \times 200 \mu \mathrm{m}^{2}$ in size and feature the adaptive gain switching amplifier with 3 stages and 352 storage cells per pixel. The prototype was read out via a chip testing box, which restricted the readout to less than 100 frames/s.

The experiment was performed at the dynamics beam line P01 (Ref. [31]) at the high-brilliance storage ring PETRA III, which is dedicated to inelastic x-ray scattering and nuclear resonant scattering. The beam line layout restricted to the most relevant components is shown

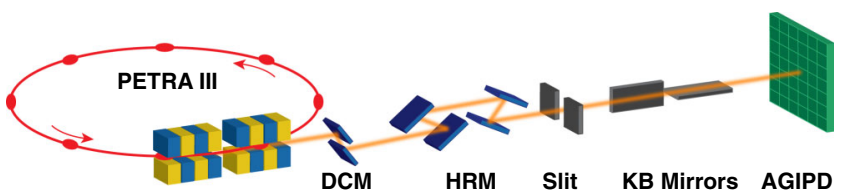

FIG. 1 (color online). Experimental setup. Synchrotron radiation is generated by the two $5 \mathrm{~m}$ undulators and is transmitted through a DCM, HRM, slit system, and set of KB mirrors. Individual synchrotron radiation pulses are recorded with the AGIPD.

schematically in Fig. 1. Two $5 \mathrm{~m} \mathrm{U32}$ undulators produced radiation at a photon energy of $14.4 \mathrm{keV}$. The radiation was monochromatized with a double-crystal monochromator (DCM) and high-resolution monochromator (HRM) positioned 48.5 and $59.9 \mathrm{~m}$ downstream from the source, respectively. The transmitted flux was about $10^{10}$ photons/s at a bandwidth of $0.9 \mathrm{meV}$ at $14.4 \mathrm{keV}$ photon energy. To reduce the number of transverse modes in the horizontal direction, a slit with a variable size was installed behind the monochromators at a distance of $61 \mathrm{~m}$ from the source. The detector was positioned in the direct beam $94 \mathrm{~m}$ downstream from the source, and intensity profiles of individual synchrotron pulses were recorded. To increase the vertical beam size as well as coherence length at the detector position a Kirkpatrick-Baez (KB) system consisting of two mirrors with a focal distance of $0.6 \mathrm{~m}$ was installed. The distance from the KB system to the detector was $3 \mathrm{~m}$, yielding a 4 times magnification of the beam size and coherence length. An increase of the transverse coherence length was required to determine the functional form of the correlation function with a higher resolution [32].

The detector was synchronized to the bunch repetition frequency of PETRA III (5.2 MHz), and about $3 \times 10^{5}$ intensity profiles of individual synchrotron pulses were recorded [see Figs. 2(a), 2(b)]. Figure 2(c) shows the average intensity profile. The beam size at the detector position was $1.4 \pm 0.15(\mathrm{~V}) \times 0.5 \pm 0.1(\mathrm{H}) \mathrm{mm}^{2} \quad(\mathrm{FWHM})$ and covered the full detector in the vertical direction. In the horizontal direction, the size of the beam was defined by the slit size of $200 \mu \mathrm{m}$ that provided an optimum balance between a low number of transverse modes and high number of registered photons. Vertical line scans of the single-pulse 2D intensity profiles [see Fig. 2(d)] were used for further analysis. The pulses have significantly different profiles due to both the chaotic nature of the radiation and the low count rates. At synchrotron sources, the latter factor dominates the intensity fluctuations due to a small degeneracy parameter, which is the average number of photons in a single mode [13].

The result of the second-order correlation function analysis [Eq. (1)] along the vertical line shown in Fig. 2 is presented in Fig. 3(a). The normalized correlation function $g^{(2)}\left(y_{1}, y_{2}\right)$ shows the expected behavior of 

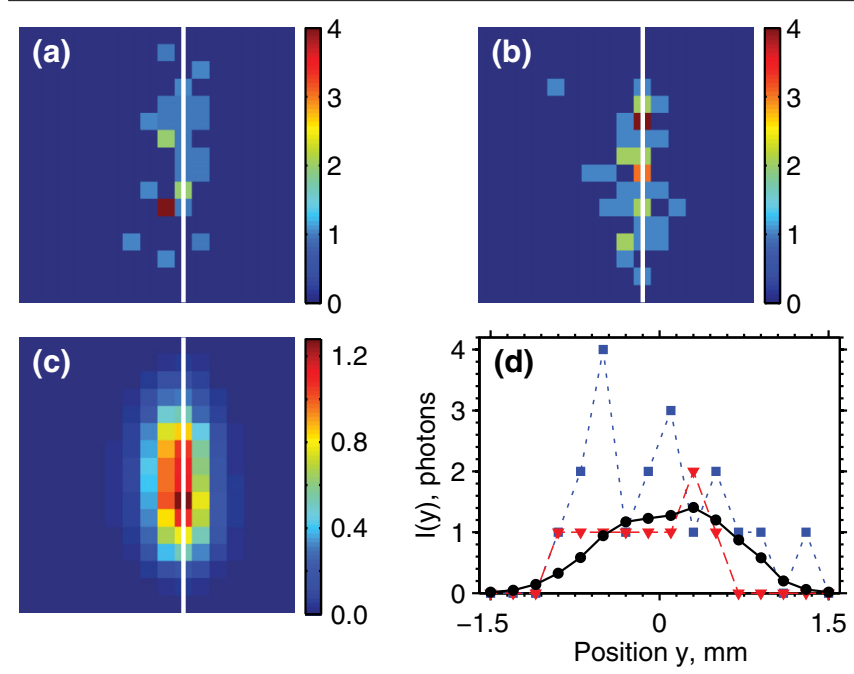

FIG. 2 (color online). Measured intensities. [(a), (b)] Typical intensity profiles of individual synchrotron radiation pulses recorded at the AGIPD. The pixel size is $200 \times 200 \mu \mathrm{m}^{2}$, and the detector size is $3.2 \times 3.2 \mathrm{~mm}^{2}$. (c) Average intensity profile. (d) Vertical line scans along white line in (a)-(c) through the typical single pulses [red triangles and dashed line (a), blue squares and dotted line (b)] and average intensity profile [black circles and solid line (c)].

maximum values for small separations between the pixels and a smooth falloff for larger separations. To determine the transverse coherence length and contrast, we extract the intensity correlation function $g^{(2)}(\Delta y), \Delta y=y_{2}-y_{1}$ from $g^{(2)}\left(y_{1}, y_{2}\right)$. Figure 3(b) shows $g^{(2)}(\Delta y)$ for three different cases: in the center of the beam and offset by $200 \mu \mathrm{m}$ (one pixel) to the left or to the right from the beam center [33]. Note that the values for both offsets are approximately equal, suggesting that the beam was quasihomogeneous as expected from the theory [34]. Because of the low flux per pulse, the values along the diagonal $y_{1}=y_{2}$ were dominated by the photon statistics and were not considered in further analysis (see the Supplemental Material [35]). The normalized correlations between neighboring pixels $y_{1}=$ $y_{2} \pm 200 \mu \mathrm{m}$ in Fig. 3(a) show higher values than expected. We attribute these high values to the parallax effect and discard these points from further analysis (Supplemental Material [35]).

The data shown in Fig. 3(b) are well reproduced by a Gaussian function fit $1+\zeta \exp \left[\left(-\Delta y^{2}\right) / l_{c}^{2}\right]$ [see Eq. (2)], which yields a transverse coherence length of $l_{c}=0.68 \pm$ $0.3 \mathrm{~mm}(\mathrm{rms})$ and a contrast value of $\zeta=1.7 \pm 0.3 \%$. The contrast is in good agreement with an estimate using PETRA III bunch parameters [36]. From the energy bandwidth of $0.9 \mathrm{meV}$ FWHM at $14.4 \mathrm{keV}$, we find the coherence time of $\tau_{c}=2 \pi / \Delta \omega \approx 4.6 \mathrm{ps}$, which together with a pulse duration of $96 \pm 3$ ps (FWHM) at normal operation conditions of PETRA III yields a contrast of $\zeta \approx 4.8 \%$ if the horizontal transverse modes are neglected. With these modes present, the expected contrast value should be lower.
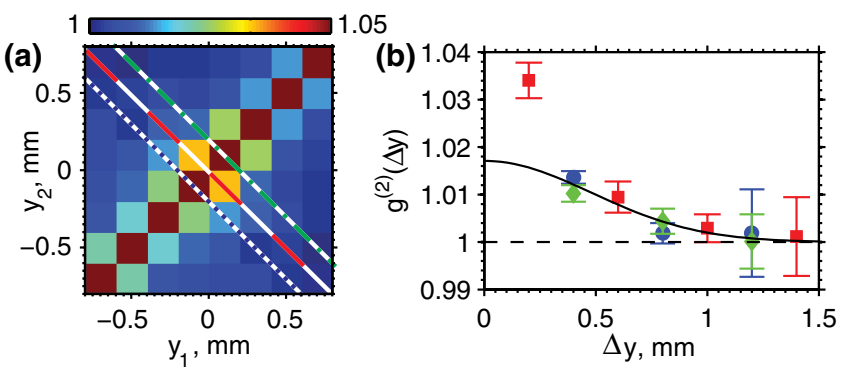

FIG. 3 (color online). Intensity correlation analysis. (a) The normalized intensity correlation function $g^{(2)}\left(y_{1}, y_{2}\right)$. (b) The normalized intensity correlation $g^{(2)}(\Delta y)$ as a function of $\Delta y$ for different cases: in the center of the beam along red dashed line in (a) (red squares), offset by a pixel to the left along blue dotted line in (a) (blue circles), and to the right along green dash-dotted line in (a) (green diamonds). Fit by a Gaussian function (black solid line) provided transverse coherence length values of $l_{c}=0.68 \pm$ $0.3 \mathrm{~mm}$ (rms) [35].

We have used the results of the transverse coherence measurements to determine the size of the synchrotron source. Using the Gaussian Schell model [13,34], where the coherence function is homogeneous across the beam and all functional dependencies are described by Gaussian functions, we found a source size of $7.8 \pm 3 \mu \mathrm{m}$ (Supplemental Material [35]). This value is in excellent agreement with the photon source size $\sigma_{y}=7.5 \mu \mathrm{m}$ estimated from the design electron beam parameters of the PETRA III storage ring $[35,36]$.

In summary, we have demonstrated successful intensity correlation measurements performed in the spatial domain with hard $\mathrm{x}$-rays at the storage ring PETRA III. These measurements allowed us to determine the transverse coherence properties of synchrotron radiation as well as to obtain a vertical photon source size of $7.8 \mu \mathrm{m}$ in excellent agreement with the value of $7.5 \mu \mathrm{m}$ determined from the electron beam parameters. The success of our measurements was based on the use of the prototype AGIPD detector that measures intensity profiles of individual synchrotron pulses with the rate of about $5 \mathrm{MHz}$. Using this pixelated detector, we were also able to record the intensity correlation function at different relative spatial separations simultaneously, which is unique compared to previous measurements. We anticipate that this technique can be extended to XFELs $[29,37,38]$ and will provide a valuable diagnostic tool for next generation $\mathrm{x}$-ray sources [20]. Because of the large frame rate, the final AGIPD detector will certainly be useful for a variety of experiments at high-brilliance x-ray sources, for example, for studies of the dynamics of matter from the nano- to microsecond time scales. We also anticipate that the unprecedented combination of outstanding temporal and spatial resolution of AGIPD will initiate new developments in the biological $[40,41]$ and materials sciences [17] at high-brilliance $\mathrm{x}$-ray sources. 
We acknowledge support of this project and fruitful discussions with E. Weckert; part of this work was supported by BMBF Proposal 05K10CHG "Coherent Diffraction Imaging and Scattering of Ultrashort Coherent Pulses with Matter" in the framework of the German-Russian collaboration "Development and Use of Accelerator-Based Photon Sources" and the Virtual Institute VH-VI-403 of the Helmholtz Association.

"Present address: University of California, San Diego, La Jolla, California 92093, USA.

singer.andrej@gmail.com

'Present address: Department of Chemistry, University of Potsdam, D-14476 Potsdam, Germany.

${ }^{\star}$ Corresponding author. Ivan.Vartaniants@desy.de

[1] R. H. Brown and R. Q. Twiss, Nature (London) 177, 27 (1956).

[2] R. H. Brown and R. Q. Twiss, Nature (London) 178, 1046 (1956).

[3] E. M. Purcell, Nature (London) 178, 1449 (1956).

[4] R. J. Glauber, Phys. Rev. 130, 2529 (1963).

[5] T. Jeltes et al., Nature (London) 445, 402 (2007).

[6] F. Arecchi, E. Gatti, and A. Sona, Phys. Lett. 20, 27 (1966).

[7] Y. Kunimune, Y. Yoda, K. Izumi, M. Yabashi, X.-W. Zhang, T. Harami, M. Ando, and S. Kikuta, J. Synchrotron Radiat. 4, 199 (1997).

[8] E. Gluskin, E. E. Alp, I. McNulty, W. Sturhahn, and J. Sutter, J. Synchrotron Radiat. 6, 1065 (1999).

[9] M. Yabashi, K. Tamasaku, and T. Ishikawa, Phys. Rev. Lett. 87, 140801 (2001).

[10] M. Yabashi, K. Tamasaku, and T. Ishikawa, Phys. Rev. Lett. 88, 244801 (2002).

[11] A. Singer et al., Phys. Rev. Lett. 111, 034802 (2013).

[12] J. W. Goodman, Statistical Optics (Wiley, New York, 1985).

[13] L. Mandel and E. Wolf, Optical Coherence and Quantum Optics (Cambridge University Press, New York, 1995).

[14] E. Ikonen, Phys. Rev. Lett. 68, 2759 (1992).

[15] For $\mathbf{r}_{1}=\mathbf{r}_{2}$, Eq. (2) contains an additional term $1 / \bar{N}$, where $\bar{N}$ is the average number of detected photons $[12,35]$. This last term is particularly important for low photon statistics.

[16] H. N. Chapman and K. A. Nugent, Nat. Photonics 4, 833 (2010).

[17] I. A. Vartanyants and O. M. Yefanov, X-ray Diffraction: Modern Experimental Techniques, edited by O. H. Seeck and B. Murphy (Pan Stanford Publishing Pte. Ltd., Singapore, 2014), Chap. 12.

[18] P. Thibault and A. Menzel, Nature (London) 494, 68 (2013).

[19] G. Grübel and F. Zontone, J. Alloys Compd. 362, 3 (2004).

[20] M. Bei et al., Nucl. Instrum. Methods Phys. Res., Sect. A 622, 518 (2010).

[21] C. Chang, P. Naulleau, E. Anderson, and D. Attwood, Opt. Commun. 182, 25 (2000).

[22] I. A. Vartanyants et al., Phys. Rev. Lett. 107, 144801 (2011).

[23] A. Singer et al., Opt. Express 20, 17480 (2012).
[24] F. Pfeiffer, O. Bunk, C. Schulze-Briese, A. Diaz, T. Weitkamp, C. David, J. van der Veen, I. Vartanyants, and I. Robinson, Phys. Rev. Lett. 94, 164801 (2005).

[25] C. Gutt et al., Phys. Rev. Lett. 108, 024801 (2012).

[26] A. Snigirev, I. Snigireva, V. Kohn, V. Yunkin, S. Kuznetsov, M. Grigoriev, T. Roth, G. Vaughan, and C. Detlefs, Phys. Rev. Lett. 103, 064801 (2009).

[27] B. Henrich et al., Nucl. Instrum. Methods Phys. Res., Sect. A 633, Suppl. 1, S11 (2011).

[28] J. Becker, D. Greiffenberg, U. Trunk, X. Shi, R. Dinapoli, A. Mozzanica, B. Henrich, B. Schmitt, and H. Graafsma, Nucl. Instrum. Methods Phys. Res., Sect. A 694, 82 (2012).

[29] M. Altarelli et al., Tech. Rep. DESY, Hamburg, Germany, 2007.

[30] J. Becker, A. Marras, A. Klyuev, F. Westermeier, U. Trunk, and H. Graafsma, JINST 8, P06007 (2013).

[31] H.-C. Wille, H. Franz, R. Röhlsberger, W. A. Caliebe, and F.-U. Dill, J. Phys. Conf. Ser. 217, 012008 (2010).

[32] We want to note here that in the horizontal direction the transverse coherence length is significantly smaller, and a larger magnification of the beam would be required to use the AGIPD for coherence measurements.

[33] To estimate the statistical errors of the measurements, we divided the measured ensemble of pulses into eleven subensembles, each consisting of $3 \times 10^{4}$ pulses, and used the spread of the resulting intensity correlation as an error measure. The pulses were randomly permuted prior to the error analysis. The uncertainties obtained from the unpermuted ensemble are larger, which suggests drifts in the beam line during the measurement.

[34] I. A. Vartanyants and A. Singer, New J. Phys. 12, 035004 (2010).

[35] See the Supplemental Material at http://link.aps.org/ supplemental/10.1103/PhysRevLett.113.064801 for the derivation of Eq. (2), estimation of the uncertainty of the transverse coherence length, determination of the source size, and the effect of the point spread function on the intensity correlation measurements.

[36] K. Balewski, W. Brefeld, W. Decking, H. Franz, R. Röhlsberger, and E. Weckert, Tech. Rep. DESY, Hamburg, Germany, 2004.

[37] P. Emma et al., Nat. Photonics 4, 641 (2010); T. Ishikawa et al., ibid. 6, 540 (2012); E. Allaria et al., ibid. 6, 699 (2012).

[38] We want to point out that single-mode optical lasers do not exhibit HBT interference. However, FELs contain several spatial and many longitudinal modes $[22,23]$ and thereby do show HBT interference [11]. This property of FEL sources is related to the basics of the self-amplified emission process [39]. As a result, FEL sources show HBT correlations similar to the synchrotron sources studied here.

[39] E. L. Saldin, E. Schneidmiller, and M. Yurkov, The Physics of Free Electron Lasers (Springer-Verlag, Berlin, 2000).

[40] A. Mancuso, O. Yefanov, and I. Vartanyants, J. Biotechnol. 149, 229 (2010).

[41] F. Stellato et al., IUCrJ 1, 204 (2014). 